\title{
Secretary Problems and Incentives via Linear Programming
}

\author{
Niv Buchbinder \\ Microsoft Research, New England \\ and \\ Kamal Jain \\ Microsoft Research, Redmond \\ and \\ Mohit Singh \\ Microsoft Research, New England
}

In the classical secretary problem an employer would like to choose the best candidate among $n$ competing candidates that arrive in a random order. Our main contribution is a new linear programming technique that we introduce as a tool for obtaining and analyzing mechanisms for the secretary problem and its variants. The linear program is formulated using judiciously chosen variables and constraints and we show a one-to-one correspondence between mechanisms for the secretary problem and feasible solutions to the linear program.

Categories and Subject Descriptors: F.2.2 [Theory of Computation]: Analysis of Algorithms and Problem Complexity - Nonnumerical Algorithms and Problems

General Terms: Algorithms, Design, Performance, Theory

Additional Key Words and Phrases: Secretary Problem, Linear Program, Duality

\section{INTRODUCTION}

In the classical secretary problem an employer would like to choose the best candidate among $n$ competing candidates that arrive in a random order. After each interview, the position of the interviewee in the total order is revealed vis-á-vis already interviewed candidates. The interviewer has to decide, irrevocably, whether to accept the candidate for the position or to reject the candidate. The objective in the basic problem is to accept the best candidate with high probability. A mechanism used for choosing the best candidate is to interview the first $n / e$ candidates for exploration, and then hire the first candidate that is better than all previous candidates. Analysis of the mechanism shows that it hires the best candidate with probability $1 / e$ and that it is optimal [Dynkin 1963; Lindley 1961].

This basic concept of $n$ elements arriving in a random order and irrevocable decisions being made by an algorithm has been explored extensively over the years. We refer the reader to the survey by Ferguson [Ferguson 1989] on the historical and extensive work on different variants of the secretary problem. Recently, there has been plenty of interest in the variants of the secretary problem and its application to the online auction problem [Hajiaghayi et al. 2004; Babaioff et al. 2008; Kleinberg

Authors' addresses: nivbuchb@microsoft.com, kamalj@microsoft.com, mohsingh@microsoft.com 
2005; Babaioff et al. 2007; Babaioff et al. 2007; Korula and Pál 2009; Babaioff et al. 2009]. Therefore, finding new ways of abstracting, as well as analyzing and designing algorithms, for secretary type problems is of major interest.

\subsection{Our Contributions}

We introduce a new linear programming technique as a tool for obtaining and analyzing mechanisms for various secretary problems [Buchbinder et al. 2009b]. We design a linear program with judiciously chosen variables and constraints and show a one-to-one correspondence between mechanisms for the secretary problem and feasible solutions to the linear program. Obtaining a mechanism which maximizes a certain objective therefore reduces to finding an optimal solution to the linear program. We use linear programming duality to give a simple proof that the mechanism obtained is optimal. We illustrate our technique by applying it to the classical secretary problem and obtaining a simple proof of optimality of the $\frac{1}{e}$ mechanism [Dynkin 1963].

Our linear program for the classical secretary problem consists of a single constraint for each position $i$, bounding the probability that the mechanism may select the $i$ th candidate. Despite its simplicity, we show that such a constraint suffices to correctly capture all possible mechanisms. Thus, optimizing over this polytope results in the optimal mechanism. The simplicity and the tightness of the linear programming formulation makes it flexible and applicable to many other variants. Capturing the set of mechanisms as a linear polytope holds the following immediate advantages.

-Computing the optimal mechanism reduces to solving a linear program.

- Proving an upper bound on the performance of any mechanism reduces to finding a feasible solution to the dual program.

- Exploring variants of the problem is as simple as adding new constraints, or manipulating the objective function of the linear program.

We next demonstrate these ideas by exploring some natural variants of the secretary problem.

Incentive Compatibility. The optimal mechanism for the classical secretary problem is to interview the first $n / e$ candidates for exploration, and then hire the first candidate that is better than all previous candidates. This mechanism suffers from a crucial drawback. The candidates arriving early have an incentive to delay their interview and candidates arriving after the position $\frac{n}{e}+1$ have an incentive to advance their interview. Such behavior challenges the main assumption of the model that interviewees arrive in a random order. This issue of incentives is of major importance especially since secretary problems have been used recently in the context of online auctions [Hajiaghayi et al. 2004; Babaioff et al. 2008].

Using the linear programming technique, we study mechanisms that are incentive compatible. We call a mechanism for the secretary problem incentive compatible if the probability of selecting a candidate at $i^{\text {th }}$ position is equal for each position $1 \leq i \leq n$. Since the probability of being selected in each position is the same, there is no incentive for any interviewee to change his or her position and therefore the interviewee arrives at the randomly assigned position. We show that there exists an 
incentive compatible mechanism which selects the best candidate with probability $1-\frac{1}{\sqrt{2}} \approx 0.29$ and that this mechanism is optimal. Incentive compatibility is captured in the linear program by introducing a set of very simple constraints.

Surprisingly, we find that the optimal incentive compatible mechanism sometime selects a candidate who is worse than a previous candidate. To deal with this issue, we call a mechanism regret-free if the mechanism only selects candidates which are better than all previous candidates. We show in the paper how to find the optimal regret free incentive compatible mechanism, as well as other interesting incentive compatible variants.

In subsequent work [Buchbinder et al. 2009a], we further explore the importance of incentive compatibility in the context of online auctions. In this context, bidders are bidding for an item and may have an incentive to change their position if this may increase their utility. We show how to obtain truthful mechanisms for such settings using underlying algorithms for secretary type problems. While there are inherent differences in the auction model and the secretary problem, a mechanism for the secretary problem is used as a building block for obtaining an incentive compatible mechanism for the online auction problem.

The $J$-choice, $K$-best Secretary Problem. We also give a linear programming formulation for a more general problem which we call the $(J, K)$-secretary problem. Here, $n$ candidates arrive randomly; the mechanism is allowed to pick up to $J$ different candidates and the objective is to pick as many as possible from the top $K$ ranked candidates. The $(1,1)$-secretary problem is the classical secretary problem. For any positive integers $J, K$, we provide a linear program which characterizes all mechanisms for the problem by generalizing the linear program for the classical secretary problem. We are also able to solve the linear programs analytically for small values of $J$ and $K$. The $(J, K)$-secretary problem is closely related to the cardinal version of the multi-secretary problem for which Kleinberg [Kleinberg 2005] gave an asymptotically tight algorithm. Better algorithms, even restricted to small values of $k$, are helpful not only for solving the original problem, but also for improving algorithms that are based upon them. For example, the secretary knapsack algorithm [Babaioff et al. 2007] uses an algorithm that is $1 / e$ competitive for maximizing the expected profit for small values of $k(k \leq 27)$.

\section{ILLUSTRATING THE TECHNIQUE: CLASSICAL SECRETARY}

In this section, we illustrate our technique by giving a simple linear program which characterizes all possible mechanisms for the classical secretary problem. We stress that the LP captures not only thresholding mechanisms, but any mechanism including probabilistic mechanisms. Hence, finding the best mechanism for the secretary problem is equivalent to finding the optimal solution to the linear program. The linear program and its dual appear in Figure 1. The following two lemmas show that the linear program exactly characterizes all feasible mechanisms for the secretary problem.

LEMma 2.1. (Mechanism to LP solution) Let $\pi$ be any mechanism for selecting the best candidate. Let $p_{i}^{\pi}$ denote the probability of selecting the candidate at position $i$. Then $p^{\pi}$ is a feasible solution to the linear program $(P)$, i.e, it satisfies the constraints $p_{i}^{\pi} \leq \frac{1}{i}\left(1-\sum_{j<i} p_{j}^{\pi}\right)$ for each $1 \leq i \leq n$. Moreover the objective 


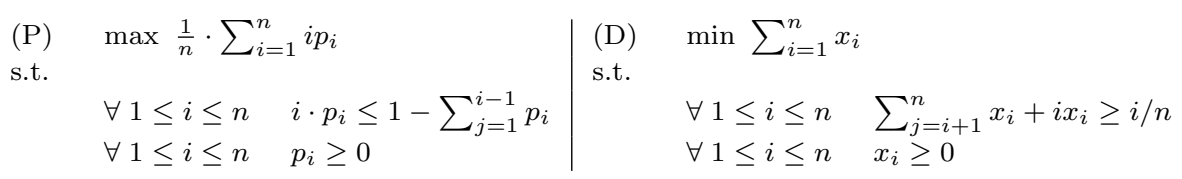

Fig. 1. Linear program and its Dual for the secretary problem

value $\frac{1}{n} \sum_{i=1}^{n} i p_{i}^{\pi}$ is at least the probability of selecting the best candidate by $\pi$.

Lemma 2.1 shows that the optimal solution to $(\mathrm{P})$ is an upper-bound on the performance of the mechanism. The following lemma shows that every LP solution actually corresponds to a mechanism which performs as well as the objective value of the solution.

LEMMA 2.2. (LP solution to Mechanism) Let $p_{i}$ for $1 \leq i \leq n$ be any feasible $L P$ solution to $(P)$. Then consider the mechanism $\pi$ which selects the candidate $i$ with probability $\frac{i p_{i}}{\left(1-\sum_{j<i} p_{j}\right)}$ if candidate $i$ is the best candidate so far and candidate $1, \ldots, i-1$ have not been selected, i.e., the mechanism reaches candidate $i$. Then $\pi$ is a mechanism which selects the best candidate with probability $\frac{1}{n} \sum_{i=1}^{n} i p_{i}$.

Using the above equivalence between LP solutions and the mechanisms, it is easy to show that the optimal mechanism can hire the best candidate with probability of no more than $1 / e$. The proof is simply by constructing a feasible solution to the dual linear program.

LEMMA 2.3 [DYNKIN 1963]. No mechanism can hire the best candidate with probability better than $1 / e$.

\section{CONCLUSION}

Characterizing the set of mechanisms in secretary type problems as a linear polytope possess many advantages. In contrast to methods of factor revealing linear programs in which a linear program is used to analyze a single algorithm, here we are able to characterize all algorithms by a linear program. One direction for future research is trying to capture more complex settings of a more combinatorial nature. One such example is the problem studied in [Babaioff et al. 2007] in which elements of a matroid arrive one-by-one. This problem seems extremely appealing since matroid constraints are exactly captured by a linear program. Another promising direction is obtaining upper bounds on performance of any mechanism for the different variants of the secretary problem. While the linear program which characterizes the performance may be too complex to obtain a simple mechanism, the dual linear may still be used for obtaining upper bounds on the performance of any algorithm.

\section{REFERENCES}

Babaioff, M., Dinitz, M., Gupta, A., Immorlica, N., And Talwar, K. 2009. Secretary problems: weights and discounts. In SODA '09: Proceedings of the Nineteenth Annual ACM -SIAM Symposium on Discrete Algorithms. Society for Industrial and Applied Mathematics, Philadelphia, PA, USA, 1245-1254.

ACM SIGecom Exchanges, Vol. 8, No. 2, December 2009. 
Babaioff, M., Immorlica, N., Kempe, D., and Kleinberg, R. 2007. A Knapsack Secretary Problem with Applications. In Proceedings of 10th International Workshop on Approximation Algorithms for Combinatorial Optimization Problems (APPROX).

Babaioff, M., Immorlica, N., Kempe, D., And Kleinberg, R. 2008. Online Auctions and Generalized Secretary Problems. SIGecom Exchange 7, 1-11.

Babaioff, M., Immorlica, N., And Kleinberg, R. 2007. Matroids, Secretary Problems, and Online Mechanisms. In Proceedings 18th ACM-SIAM Symposium on Discrete Algorithms.

Buchbinder, N., Singh, M., And Jain, K. 2009a. Incentives in Online Auctions and Secretary Problems via Linear Programming. In Manuscript.

Buchbinder, N., Singh, M., And Jain, K. 2009b. Secretary Problems via Linear Programming. In Manuscript.

Dynkin, E. B. 1963. The Optimum Choice of the Instant for Stopping a Markov Process. Sov. Math. Dokl. 4.

Ferguson, T. S. 1989. Who Solved the Secretary Problem? Statist. Sci. 4, 282-289.

Hajiaghayi, M. T., Kleinberg, R., and Parkes, D. C. 2004. Adaptive Limited-Supply Online Auctions. In Proceedings of the 5th ACM Conference on Electronic Commerce.

Kleinberg, R. 2005. A Multiple-Choice Secretary Algorithm with Applications to Online Auctions. In Proceedings of the Sixteenth Annual ACM-SIAM Symposium on Discrete algorithms.

Korula, N. AND PÁL, M. 2009. Algorithms for secretary problems on graphs and hypergraphs. In $\operatorname{ICALP}$ (2). 508-520.

Lindley, D. V. 1961. Dynamic Programming and Decision Theory. Applied Statistics 10, 39-51. 